University of Warwick institutional repository: http://go.warwick.ac.uk/wrap This paper is made available online in accordance with publisher policies. Please scroll down to view the document itself. Please refer to the repository record for this item and our policy information available from the repository home page for further information.

To see the final version of this paper please visit the publisher's website. Access to the published version may require a subscription.

Author(s): K. G. McCLEMENTS, R. O. DENDY, M. E. DIECKMANN, A. YNNERMAN and S. C. CHAPMAN

Article Title: Surfatron and stochastic acceleration of electrons in astrophysical plasmas

Year of publication: 2005

Link to published

version: http://dx.doi.org/10.1017/S0022377804003514

Publisher statement: None 


\title{
Surfatron and stochastic acceleration of electrons in astrophysical plasmas
}

\author{
K. G. M c CLEMEN T S ${ }^{1}$, R. O. DE N D Y ${ }^{1}$, M. E. D I E CK M A N N ${ }^{2}$, \\ A. Y N N R M A N ${ }^{2}$ and S.C. CHA P M A N 3 \\ ${ }^{1}$ UKAEA Culham Division, Culham Science Centre, Abingdon, \\ Oxfordshire OX14 3DB, UK \\ email: k.g.meclements@ukaea.org.uk \\ ${ }^{2}$ Department of Science and Technology, Linköping University, Campus Norrköping, \\ 60174 Norrköping, Sweden \\ ${ }^{3}$ Department of Physics, University of Warwick, Coventry CV4 7AL, UK
}

(Received 9 September 2004)

\begin{abstract}
Electron acceleration by large amplitude electrostatic waves in astrophysical plasmas is studied using particle-in-cell (PIC) simulations. The waves are excited initially at the electron plasma frequency $\omega_{\text {pe }}$ by a Buneman instability driven by ion beams: the parameters of the ion beams are appropriate for high Mach number astrophysical shocks, such as those associated with supernova remnants (SNRs). If $\omega_{\text {pe }}$ is much higher than the electron cyclotron frequency $\Omega_{\mathrm{e}}$, the linear phase of the instability does not depend on the magnitude of the magnetic field. However, the subsequent time evolution of particles and waves depends on both $\omega_{\mathrm{pe}} / \Omega_{\mathrm{e}}$ and the size of the simulation box $L$. If $L$ is equal to one wavelength, $\lambda_{0}$, of the Buneman-unstable mode, electrons trapped by the waves undergo acceleration via the surfatron mechanism across the wave front. This occurs most efficiently when $\omega_{\mathrm{pe}} / \Omega_{\mathrm{e}} \simeq 100$ : in this case electrons are accelerated to speeds of up $c / 2$ where $c$ is the speed of light. In a simulation with $L=4 \lambda_{0}$ and $\omega_{\mathrm{pe}} / \Omega_{\mathrm{e}}=100$, it is found that sideband instabilities give rise to a broad spectrum of wavenumbers, with a power law tail. Some stochastic electron acceleration is observed in this case, but not the surfatron process. Direct integration of the electron equations of motion, using parameters approximating to those of the wave modes observed in the simulations, suggests that the surfatron is compatible with the presence of a broad wave spectrum if $\omega_{\mathrm{pe}} / \Omega_{\mathrm{e}}>100$. It is concluded that a combination of stochastic and surfatron acceleration could provide an efficient generator of mildly relativistic electrons at $\mathrm{SNR}$ shocks.
\end{abstract}

\section{Introduction}

Radio synchrotron emission from supernova remnants (SNRs) indicates the presence of highly relativistic electrons in such objects (Biermann and Cassinelli 1993). The diffusive shock mechanism (Bell 1978) provides an efficient means of accelerating electrons at SNR shocks from a mildly relativistic threshold, but does not solve the problem of raising electrons to this threshold. A possible solution is provided

$\dagger$ Current address: Theoretische Physik IV, Ruhr-Universität Bochum, D-44780 Bochum, Germany. 
by large amplitude electrostatic waves, whose efficacy in producing energetic electrons has recently been demonstrated in laboratory experiments (see the review by Bingham et al. 2004). Such waves provide the required free energy for the 'surfatron' process, proposed by Katsouleas and Dawson (1983) as a laser-plasma accelerator that can in principle produce electrons of arbitrarily high energy. It has been difficult to exploit fully the potential of the surfatron in laboratory experiments, which are necessarily restricted in size. However, the large spatial scales of astrophysical plasmas such as those in the vicinity of SNR shocks suggest that the surfatron should be examined as a possible source of energetic particles in such plasmas. Ions reflected from SNR shocks can excite large amplitude electrostatic waves, which are an essential ingredient of the surfatron.

There is increasing interest in the possibility that electrons could be accelerated in astrophysical plasma environments by large amplitude electrostatic waves. Dieckmann et al. (2000a, b) and McClements et al. (2001) used a particle-in-cell (PIC) code to study, in one space dimension, wave instabilities excited by ion beams at SNR shocks. The magnetic field $B$ was set equal to zero in the simulations described by Dieckmann et al. (2000b): the ion beams in this case were observed to excite electrostatic, Buneman-type instabilities at frequencies $\omega$ close to the electron plasma frequency $\omega_{\text {pe }}$. In earlier magnetized simulations (Dieckmann et al. 2000a), with the ratio of electron plasma frequency $\omega_{\text {pe }}$ to electron cyclotron frequency $\Omega_{\mathrm{e}}$ set equal to 10 and the ion beams propagating perpendicular to the magnetic field, it was found that the instability was still Buneman-like if the ion beam drift speed was at least several times the initial electron thermal speed $v_{\mathrm{e}}$. In the Buneman regime it was found that the instability invariably saturated because of electron trapping by the wave. The subsequent evolution of the wave depended on the number of wavelengths $\lambda_{0}$ of the initial Buneman instability contained in the simulation box, and the initial ratio of bulk ion temperature $T_{\mathrm{i}}$ to electron temperature $T_{\mathrm{e}}$. Periodic boundary conditions were used, which meant that the only wave modes that could be represented had wavenumbers $k=2 \pi n / L$ where $L$ is the box size and $n$ is an integer. With $L=\lambda_{0}$ and $T_{\mathrm{i}} / T_{\mathrm{e}}=1$, it was found that the initial wave at $\omega_{\text {pe }}$ collapsed into low frequency (ion acoustic) waves and back-scattered waves at $\omega \simeq \omega_{\text {pe }}$. With the same simulation box size and $T_{\mathrm{i}} / T_{\mathrm{e}}=100$, the wave at $\omega_{\text {pe }}$ was eventually replaced with two other waves in a similar frequency range supported by trapped electrons: at least one of these appears to have been a BernsteinGreene-Kruskal (BGK) mode (Bernstein et al. 1957). When $L$ was set equal to $4 \lambda_{0}$ and $T_{\mathrm{i}} / T_{\mathrm{e}}=100$, on the other hand, it was found that the initial Buneman wave collapsed soon after reaching its saturation amplitude, apparently because of sideband instabilities (Kruer et al. 1969). In a real plasma, trapped electron modes such as those observed in the simulation with $L=\lambda_{0}, T_{\mathrm{i}} / T_{\mathrm{e}}=100$ are expected to exist for a finite time determined principally by the initial wave amplitude (Brunetti et al. 2000). Simulations that exclude wavevectors other than those of a linearly unstable mode and multiples thereof are somewhat artificial, but serve two useful purposes: first, they provide a link between theory and real plasmas; and second, they make it possible to study individual nonlinear plasma physics processes that may be difficult to observe otherwise.

Strong electron acceleration was observed by Dieckmann and co-workers in magnetized simulations (Dieckmann et al. 2000a; McClements et al. 2001), but not in unmagnetized simulations (Dieckmann et al. 2000b). The acceleration of charged particles in the presence of a large amplitude electrostatic wave (of the type 
excited by a Buneman instability) and a magnetic field can be either regular, as in the surfatron (Katsouleas and Dawson 1983), or stochastic; an example of the latter process is provided by lower hybrid wave heating of ions in tokamaks (Karney 1978). There is clear evidence for a surfatron acceleration phase in PIC simulations of electron injection in the foot region of high Alfvénic Mach number SNR shocks by Schmitz et al. (2002b). We refer in particular to their Fig. 7: this displays extended episodes of linear growth in the electron velocity component perpendicular to the magnetic field and the shock normal, during a time that the electrons are observed (see Figs 5 and 6 of Schmitz et al. 2002b) to be trapped in nonlinear electrostatic field structures. In most astrophysical plasma contexts it is likely that interplay between several physical processes is ultimately responsible for particle energization. In the present paper, we focus initially on the surfatron mechanism in a well defined wave. It will be seen that this treatment naturally leads on to the consideration of multiple transient surfatron acceleration events - an essentially stochastic process.

In the next section we summarize the theory of the surfatron, pointing out its limitations and the need to model it self-consistently using the PIC approach. The PIC simulation results are presented in Sec. 3 and interpreted (with the aid of direct numerical solutions of the electron equations of motion) in Sec. 4 . The results are summarized and their astrophysical implications discussed in Sec. 5.

\section{Surfatron mechanism}

The surfatron was proposed originally by Katsouleas and Dawson (1983) as a laserplasma acceleration scheme. They considered an electrostatic wave $E=E_{0} \sin (k x-$ $\omega t$ ), excited, for example, by a pair of short-pulse lasers via the beat wave mechanism (Tajima and Dawson 1979), propagating in the $x$-direction perpendicular to a steady magnetic field $\mathbf{B}$ in the $z$-direction (see Fig. 1). An electron with $v_{x}$ approximately equal to the wave phase speed $v_{\phi}=\omega / k$ can be trapped by the wave. Transforming to the frame in which the wave has zero phase speed, one infers an electric field in the $y$-direction

$$
E_{y}^{\prime}=\gamma_{\phi}\left(E_{y}-v_{\phi} B\right)=-\gamma_{\phi} v_{\phi} B,
$$

where $\gamma_{\phi}=\left(1-v_{\phi}^{2} / c^{2}\right)^{-1 / 2}, c$ being the speed of light. We use primes to denote quantities in the wave frame $\left(E_{y}=0\right.$, since the wave is purely electrostatic and is propagating in the $x$-direction). The $x$-component of the force on the electron in the wave frame is

$$
F_{x}^{\prime}=-e\left[E_{0} \sin \left(k x^{\prime} / \gamma_{\phi}\right)+\gamma_{\phi} v_{y}^{\prime} B\right] .
$$

The first term on the right-hand side has a trapping effect on the electron; the second (Lorentz force) term has a de-trapping effect. A sufficient condition for the electron to undergo continuous acceleration in the $y$-direction is that it remain trapped. Since $v_{y}^{\prime}<c$, trapping will continue indefinitely if

$$
E_{0}>\gamma_{\phi} c B \text {. }
$$

On the assumption that this condition is satisfied, previous authors (Katsouleas and Dawson 1983; Mendonça 1996) have proceeded to consider the case of deeplytrapped electrons, which remain close to the bottom of the wave potential well. In such circumstances $v_{x}$ is approximately equal to $v_{\phi}$ at all times. Setting $v_{x}=v_{\phi}$ in the Lorentz force equation, one obtains trivially the following solution for $v_{y}$ 


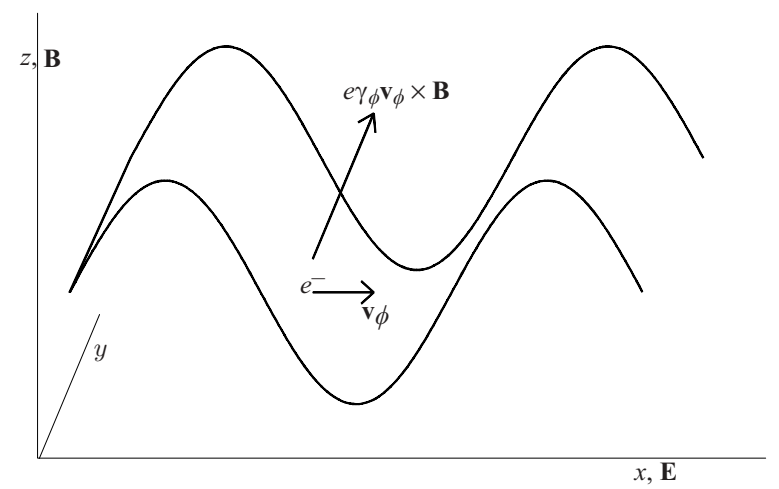

Figure 1. Geometry of surfatron acceleration. An electron with $v_{x}$ approximately equal to the phase speed $v_{\phi}$ of a large amplitude electrostatic wave is trapped by the wave, and is subject to a force in the $y$-direction equal to $e \gamma_{\phi} v_{\phi} B$, where $\gamma_{\phi}=\left(1-v_{\phi}^{2} / c^{2}\right)^{-1 / 2}$.

(Katsouleas and Dawson 1983):

$$
v_{y}=\frac{v_{\phi} \Omega_{\mathrm{e}} t}{\gamma_{\phi}\left(1+\Omega_{\mathrm{e}}^{2} t^{2} v_{\phi}^{2} / c^{2}\right)^{1 / 2}},
$$

It is straightforward to verify that $v=\left(v_{x}^{2}+v_{y}^{2}\right)^{1 / 2} \rightarrow c$ as $t \rightarrow \infty$, so that arbitrarily high electron energies can in principle result from this process. For $v_{\phi} \ll c$, (4) indicates that an initially non-relativistic electron is accelerated to relativistic energies in a timescale $\tau_{\text {acc }} \sim c /\left(v_{\phi} \Omega_{\mathrm{e}}\right) \propto 1 / B$.

Several factors can limit the effectiveness of surfatron acceleration in practice. For example, the above analysis assumes that the wave is uniform and of infinite extent in the $y$-direction. It also assumes that the wave is not affected by the electron population. Even without the presence of a magnetic field, large amplitude electrostatic waves undergo Landau damping in the presence of Maxwellian electron populations, regardless of their initial amplitude. As we noted in Sec. 1, both PIC (Dieckmann et al. 2000b) and Vlasov (Brunetti et al. 2000) simulations with box size $L$ greater than one wavelength indicate that the lifetime of a monochromatic electrostatic wave is limited by the excitation of sideband instabilities. Since surfatron acceleration relies on particle trapping, it is effective only for as long as the wave amplitude is high enough for a significant number of electrons to remain trapped by it. For a wave with a given amplitude and lifetime, (3) and (4) imply that there is an optimum value of the magnetic field for surfatron acceleration to be effective: $B$ must be low enough that electrons remain trapped, but high enough that they undergo significant acceleration within the lifetime of the wave. A further complication is that (4) is only strictly applicable to deeply trapped electrons, for which $v_{x}$ is always close to $v_{\phi}$. In fact, the unmagnetized PIC simulations of Dieckmann et al. (2000b) indicate that trapped electrons generally lie close to the separatrix of the trapped-particle island, and that the width of the island in velocity space is comparable to $v_{\phi}$. In these circumstances, one cannot treat $v_{x}$ as a constant and (4) is therefore not strictly valid.

The purpose of the simulations described in the following section is to investigate the surfatron process in as self-consistent a manner as possible. In particular, the PIC method makes it possible to study the effects of trapped and accelerated electrons on the evolution of the waves. 


\section{Simulations}

\subsection{Initial parameters}

Except for the magnetic field, which we set equal to $3.5 \times 10^{-8} \mathrm{~T}$, the parameters are identical to those of the unmagnetized simulations described by Dieckmann et al. (2000b). A realistic ion/electron mass ratio (1836) is used. Periodic boundary conditions are imposed on the one-dimensional simulation box, which has 90 cells (each of length $2.04 \mathrm{~m}$ ) in a simulation with $L=\lambda_{0}$, and 360 cells in a simulation with $L=4 \lambda_{0}$. In the case of $L=\lambda_{0}$, the electrons are represented by more than $10^{5}$ particles per cell. The electrons initially have a Maxwellian distribution with temperature $T_{\mathrm{e}} \simeq 9 \mathrm{eV}$, and density $n_{\mathrm{e}} \simeq 1.2 \times 10^{8} \mathrm{~m}^{-3}$. In general, temperatures and densities upstream of SNR shocks are difficult to estimate. However, observations of certain regions close to the supernova SN1987A (Blondin and Lundquist 1993), for example, suggest values of $T_{\mathrm{e}}$ and $n_{\mathrm{e}}$ that are broadly consistent with those quoted above. Estimates of $B$ at SNRs are even less certain than those of density and temperature. Achterberg and Ball (1994) have inferred minimum field values upstream of the shock associated with SN1978K in the range $2.5 \times 10^{-10} \mathrm{~T}$ to $2.5 \times 10^{-8} \mathrm{~T}$. The magnetic field assumed in our simulations is close to the upper end of this range. It should be stressed, however, that the simulation results appear to depend specifically on the ratio $\omega_{\mathrm{pe}} / \Omega_{\mathrm{e}}$ rather than $n_{\mathrm{e}}$ and $B$ individually.

The ion (proton) population in each simulation consists initially of a Maxwellian bulk component, with a temperature of $900 \mathrm{eV}$, and two beams, propagating in opposite directions at speeds $v_{\mathrm{b}}=0.06 \mathrm{c}$, perpendicular to the magnetic field. The beams also have Maxwellian distributions, with temperatures equal to $900 \mathrm{eV}$ and $150 \mathrm{keV}$. The existence of well-defined ion beams in the immediate upstream region of high Mach number quasi-perpendicular shocks is clearly indicated by space plasma observations (Sckopke et al. 1983), hybrid simulations (Quest 1986), and full particle simulations (Shimada and Hoshino 2000). We choose the beams in our simulations to have mean velocities equal in magnitude and opposite in direction in order to ensure zero current; the use of unequal beam temperatures means that only one beam gives rise to a strong Buneman instability (Dieckmann et al. 2000b). The number density of each beam is $n_{\mathrm{e}} / 6$, giving a total beam concentration that is comparable to reflected ion fractions observed in hybrid simulations of high Mach number shocks (Quest 1986). Such simulations indicate that the reflected ion speed in the upstream plasma frame is of the order of the shock speed, $v_{\mathrm{s}}$. Thus, our simulations are applicable to shocks with $v_{\mathrm{s}} \sim 0.06 c$ : it has been inferred observationally that efficient electron acceleration only occurs at astrophysical shocks with $v_{\mathrm{s}}$ exceeding a critical value of about $0.03 c$ (Biermann and Cassinelli 1993).

Wavenumbers, $k$, are normalized to $2 \pi / \lambda_{0}$, where $\lambda_{0}=183.6 \mathrm{~m}$ is approximately equal to $2 \pi v_{\mathrm{b}} / \omega_{\text {pe }}$, the expected wavelength of the Buneman-unstable mode (Dieckmann et al. 2000a). The wave amplitude of a mode with dimensionless wavenumber $\tilde{k}=k \lambda_{0} / 2 \pi$ is computed by Fourier transforming the electric field $E(x, t)$ over the space variable $x$ :

$$
E_{0}(\tilde{k}, t)=\frac{2}{N}\left|\sum_{n=0}^{N-1} E\left(x_{n}, t\right) \exp \left[-\frac{2 \pi i \tilde{k} x_{n}}{L}\right]\right|
$$

where $N$ is the number of cells and $x_{n}$ is the position of the $n$th cell. It is easily verified that the summation in (5) gives the true amplitude of a sinusoidal wave. 

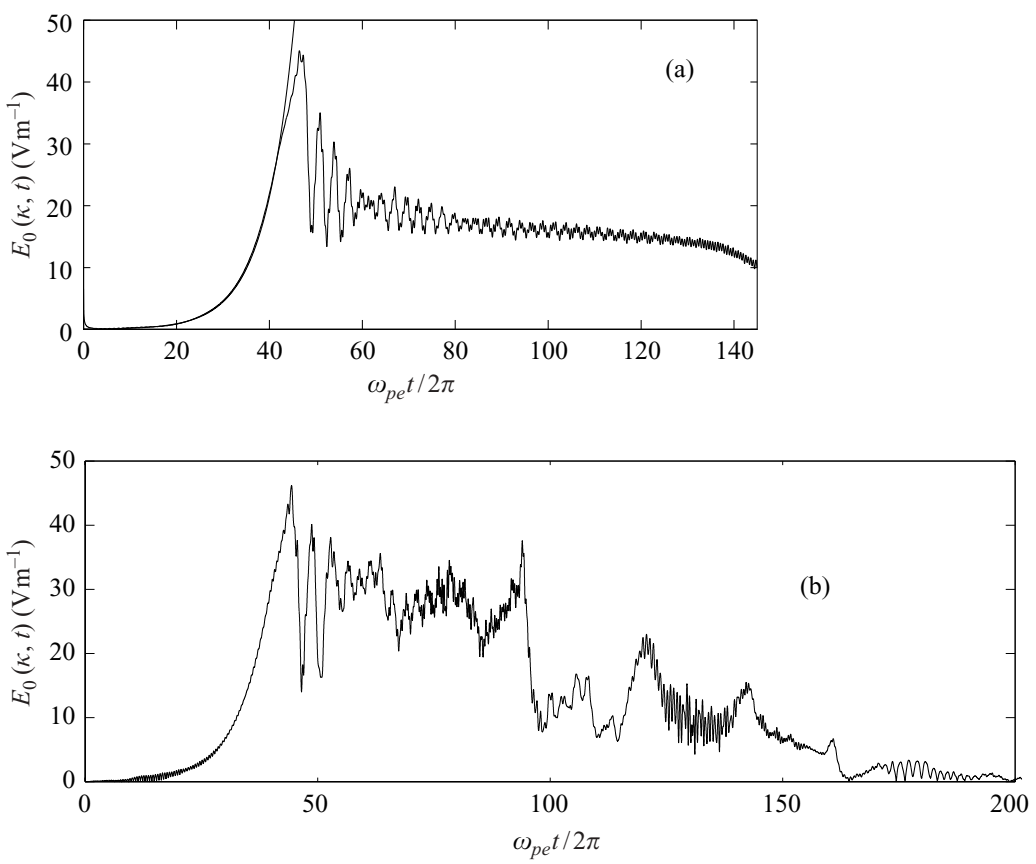

Figure 2. Amplitude of waves with wavelength $\lambda$ equal to the predicted Buneman wavelength $\lambda_{0} \simeq 2 \pi v_{\mathrm{b}} / \omega_{\mathrm{pe}}$ in (a) unmagnetized and (b) magnetized simulations with box size $L=\lambda_{0}$ (after Dieckmann et al. 2000b and McClements et al. 2001).

\subsection{Simulation with $L=\lambda_{0}$}

As in the unmagnetized simulations (Dieckmann et al. 2000b), it was found that the ion beam propagating in the positive $x$-direction excited a Buneman instability at $\omega \simeq \omega_{\text {pe }}, \tilde{k}=1$. We begin by comparing, in Fig. 2, the time evolution of the Buneman-unstable mode (a) without and (b) with the magnetic field present. The linear phase of the instability is identical in the two cases: the wave undergoes a phase of exponential growth up to about $45 \mathrm{~V} \mathrm{~m}^{-1}$. After some large amplitude oscillations, the two waves start to evolve in different ways. In the unmagnetized case, the wave falls to about $20 \mathrm{~V} \mathrm{~m}^{-1}$, where it remains for about 80 plasma periods, before declining further. In the magnetized case, the wave fluctuates around a somewhat higher amplitude (about $30 \mathrm{~V} \mathrm{~m}^{-1}$ ), but then collapses to about $10 \mathrm{~V} \mathrm{~m}^{-1}$ at $\omega_{\mathrm{pe}} t / 2 \pi \simeq 95$. Thereafter it remains at a similar amplitude before collapsing again at $\omega_{\text {pe }} t / 2 \pi \simeq 160$. It is clear from these plots that the magnetic field has a significant influence on the nonlinear evolution of the wave.

Snapshots of the electron distribution in $\left(v_{x}, v_{y}\right)$ space during the magnetized simulation are shown in Fig. 3. The three time frames are (a) just before the wave collapse at $\omega_{\text {pe }} t / 2 \pi \simeq 95$, (b) just after the wave collapse, and (c) at the end of the simulation. The most distinctive feature in Fig. 3(a) is a sharp-edged, approximately rectangular-shaped structure at $v_{x} \gtrsim 0, v_{y}>0$. Electrons in this region of velocity space are trapped by the wave, whose phase speed $v_{\phi} \simeq v_{\mathrm{b}}=0.06 \mathrm{c}$ defines a line through the centre of the rectangle parallel to the $v_{y}$ axis. The fact that the rectangle extends along the $v_{y}$ axis is due to surfatron acceleration: the maximum value of $v_{y}$ is about $c / 4$, which is consistent with (4) when $v_{\phi}=0.06 c$ and $\omega_{\text {pe }} t / 2 \pi$ is set equal to the lifetime of the wave at that point of the simulation (cf. Fig. $2(b)$ ). 

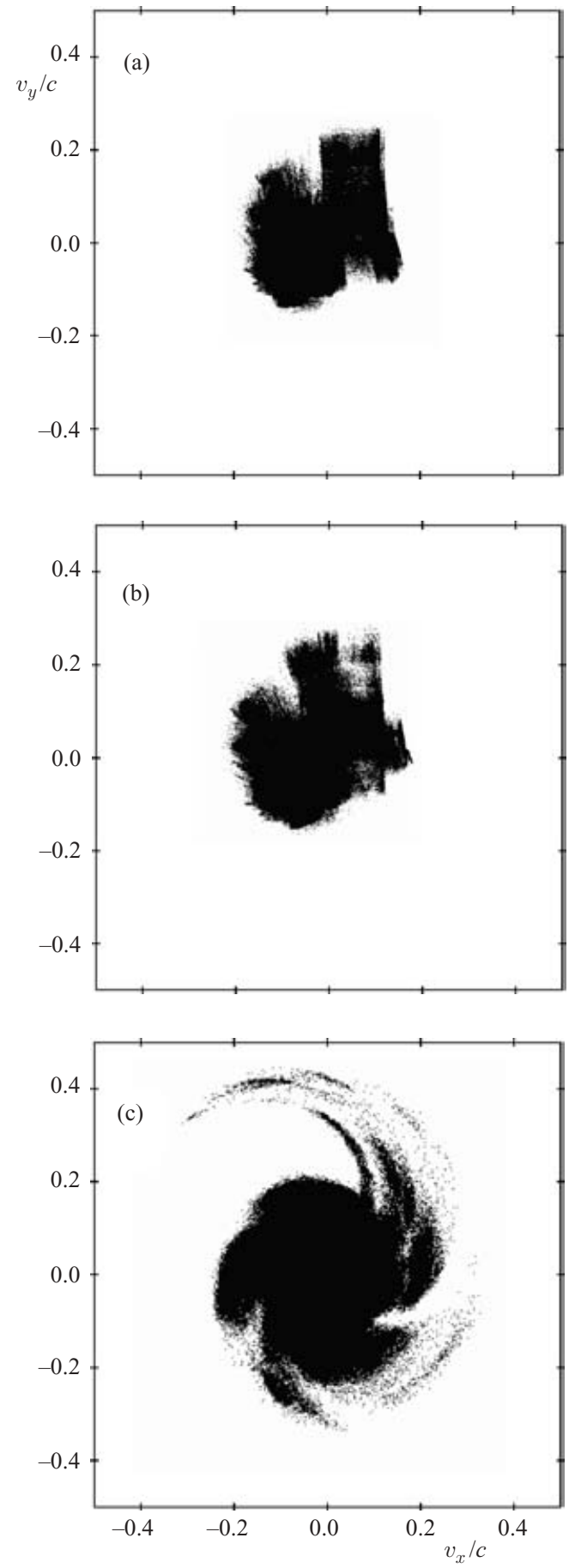

Figure 3. Electron velocity distribution perpendicular to the magnetic field in the magnetized simulation with $L=\lambda_{0}$ : (a) just before the collapse in wave amplitude at $\omega_{\mathrm{pe}} t / 2 \pi \simeq 95 \mathrm{in}$ Fig. 2(b); (b) just after the wave collapse; and (c) at the end of the simulation. 
The width of the rectangle in $v_{x}$ is determined by the wave amplitude $E_{0}$, since all electrons with $\left|v_{\phi}-v_{x}\right|$ less than a critical value proportional to $E_{0}^{1 / 2}$ are trapped, and can thus undergo surfatron acceleration. These electrons oscillate in $v_{x}$, with an amplitude which tends to zero as $v$ approaches $c$. In the sub-relativistic regime of Fig. 3(a), the amplitude is only weakly dependent on $v_{y}$.

When the wave collapses at $\omega_{\text {pe }} t / 2 \pi \simeq 95$, the trapped region of velocity space greatly diminishes in size. Electrons close to the original trapped-particle island separatrix that cease to be trapped gyrate in the magnetic field, and stop accelerating. This can be seen in Fig. 3(b). Part of the rectangular structure noted in Fig. 3(a) has broken away, and is beginning a slow anti-clockwise rotation in the $\left(v_{x}, v_{y}\right)$ plane. A significant number of electrons remain trapped, and undergo further surfatron acceleration, although the long-term fall in wave amplitude means that the trapped electron population continues to decline. By the end of the simulation [Fig. 3(c)], the combination of a gradual diminution of the trapping electric field, gyration in the magnetic field and stochastic acceleration (cf. Dieckmann et al. 2000a) gives rise to a velocity space distribution resembling a spiral galaxy.

Plots of the electron $\left(x, v_{x}\right)$ phase space (not shown here) show results similar to those obtained in the unmagnetized simulations reported by Dieckmann et al. (2000b): as the wave grows exponentially, a trapped-particle island can be seen forming. Most of the trapped electrons remain close to the island separatrix, with only a few particles migrating towards the centre. The proximity of trapped electrons to the separatrix means that they are detrapped by relatively small reductions in the wave amplitude.

\subsection{Simulation with $L=4 \lambda_{0}$}

In this simulation the linear phase of the instability, up to $\omega_{\mathrm{pe}} t / 2 \pi \simeq 40$, is similar to that shown in Fig. 2. As the amplitude of the initial Buneman wave at $\tilde{k}=1$ builds up, harmonics of this wave appear, with wavenumbers up to $\tilde{k}=19$. After $\omega_{\text {pe }} t / 2 \pi \simeq 40$, the initial wave and its harmonics are replaced with a continuum: waves appear at every $\tilde{k}$ permitted by the finite size of the simulation box. A similar phenomenon has been observed in both PIC (Dieckmann et al. 2000b) and Vlasov (Brunetti et al. 2000) simulations: it can be attributed to the excitation of sideband instabilities, initially at $\tilde{k}=0.75,1.25$, and subsequently at other wavenumbers. A snapshot of the continuous spectrum is shown in Fig. 4. Approximating the high wavenumber tail of the distribution by a power law, with $E_{0} \propto \tilde{k}^{-\alpha}$, we obtain $\alpha \simeq 2.3$. At $0.25 \leqslant \tilde{k} \lesssim 1$, where the spectrum departs from a power law, there are several modes of comparable amplitude, at or slightly below $10 \mathrm{~V} \mathrm{~m}^{-1}$. These modes have $\omega \sim \omega_{\text {pe }}$, and therefore the phase speed of the longest wavelength mode $(\tilde{k}=0.25)$ is $v_{\phi} \sim 4 \omega_{\text {pe }} \lambda_{0} / 2 \pi \simeq 0.24 c$.

The electron $\left(x, v_{x}\right)$ phase space at $\omega_{\text {pe }} t / 2 \pi \simeq 43$, shortly after the appearance of the continuous $\tilde{k}$ spectrum, is shown in Fig. 5. In contrast to the single trappedparticle island found in the simulation with $L=\lambda_{0}$, there is a complex interaction between several such islands, resulting in the formation of irregularly-shaped phase space structures. Strong surfatron acceleration was not observed, but it is significant that the $v_{x}$ distribution in Fig. 5 extends approximately up to the phase speed of the fastest-propagating mode in the system, with $\tilde{k}=0.25$. Figure 5 indicates acceleration of electrons in $v_{x}$ (rather than $v_{y}$, as in the surfatron) due to what appears to be an essentially random interaction with several high amplitude wave 


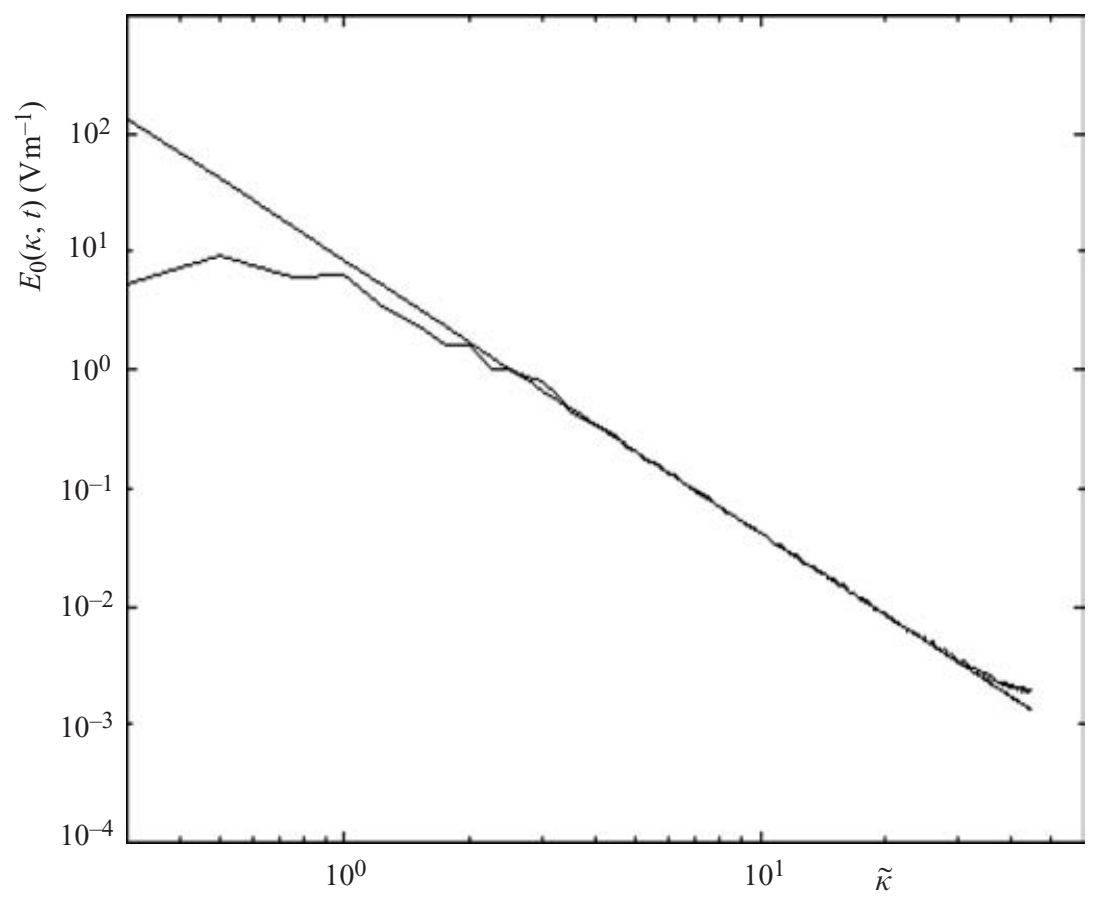

Figure 4. Snapshot of wave spectrum in simulation with $L=4 \lambda_{0}$, after $\omega_{\text {pe }} t / 2 \pi \simeq 40$. The straight line indicates a power law fit to the high $\tilde{k}$ tail of the spectrum, with index $\alpha \simeq 2.3$.

modes. This can be a rapid process: electrons were observed to accelerate from $0.14 c$ to $0.25 c$ in five plasma periods (i.e. a twentieth of one electron cyclotron period). The waves in the high $\tilde{k}$ tail of the spectrum do not appear to play a strong role in the acceleration process: in a simulation with fewer beam ions per cell (512 rather than 3200), the asymptotic slope of the spectrum was found to be slightly different ( $\alpha \simeq 2$ rather than 2.3), but the electrons behaved in a similar fashion.

\section{Interpretation}

In order to understand the phase space plots in Figs 3 and 5 it is instructive to solve directly the equations of motion for single electrons, using wave parameters corresponding approximately to the simulation results shown in Figs 2 and 4 . In the case of the simulation with $L=\lambda_{0}$, there is essentially only one wave mode in the system, and the equations of motion are those considered by Katsouleas and Dawson (1983):

$$
\begin{gathered}
\frac{d}{d t}\left(\gamma v_{x}\right)=-\frac{e E_{0}}{m_{\mathrm{e}}} \sin (k x-\omega \mathrm{t})-\Omega_{\mathrm{e}} v_{y}, \\
\frac{d}{d t}\left(\gamma v_{y}\right)=\Omega_{\mathrm{e}} v_{x},
\end{gathered}
$$

where $\gamma=\left[1-\left(v_{x} / c\right)^{2}-\left(v_{y} / c\right)^{2}\right]^{-1 / 2}$ is the electron Lorentz factor.

Numerical solutions of $(6)$ and $(7)$ for parameters corresponding approximately to those of the simulation with $L=\lambda_{0}$ are shown in Fig. 6. The wave amplitude, assumed to be constant, is taken to be $30 \mathrm{~V} \mathrm{~m}^{-1}$ [cf. Fig. 2(b)]. The solid curve 


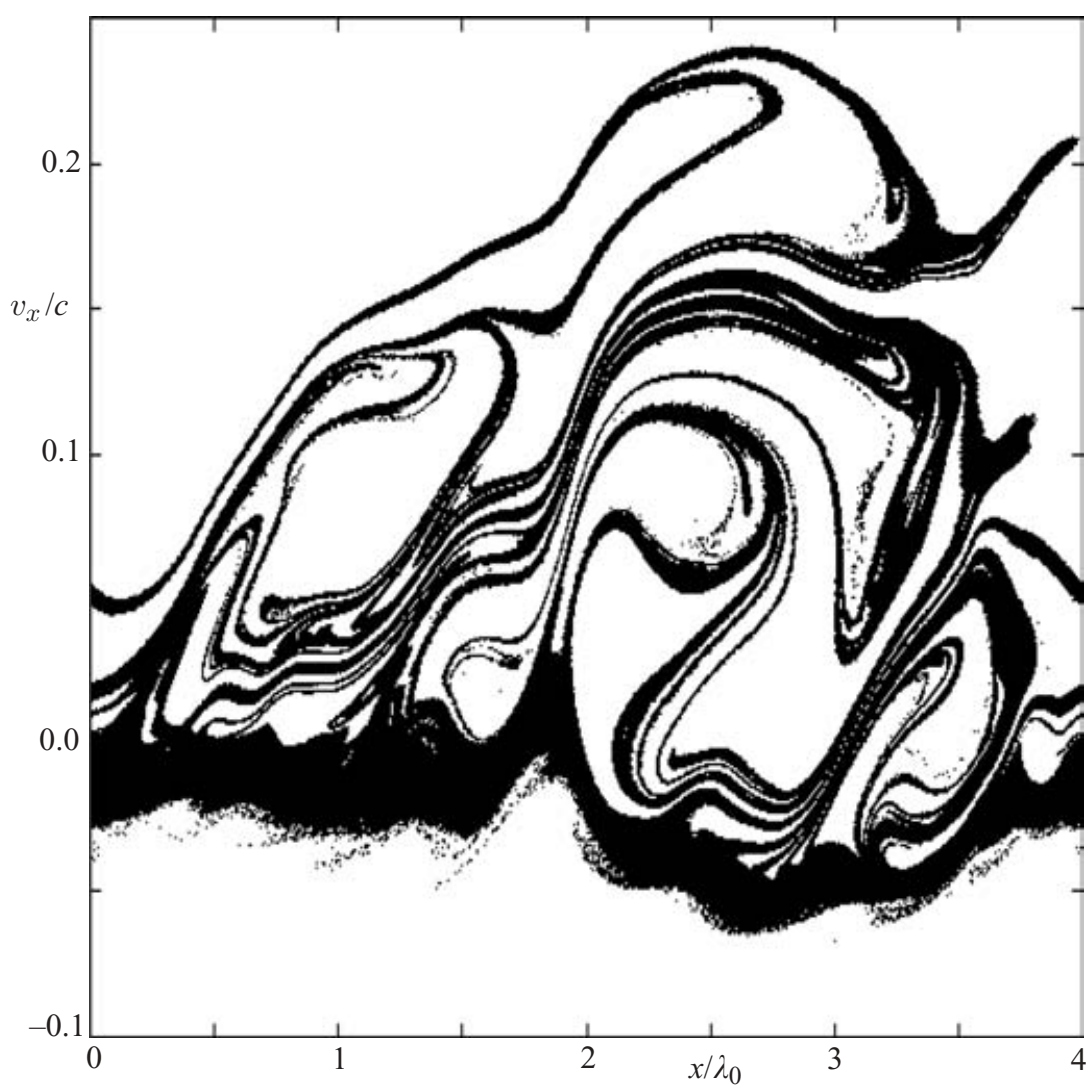

Figure 5. Electron distribution in $\left(x, v_{x}\right)$ space at $\omega_{\text {pe }} t / 2 \pi=43$ in simulation with $L=4 \lambda_{0}$ (after McClements et al. 2001).

indicates $v_{y}(t)$ for a trapped electron initially lying close to the separatrix of the trapped-particle island $\left(x=0, v_{x 0}=0.12 c\right)$; the short dashed curve, which coincides almost exactly with the solid curve, is the solution for an electron initially at the centre of the island $\left(x=0, v_{x 0}=0.06 c\right)$; the long dashed curve is the solution for an untrapped electron, initially just outside the separatrix. The two trapped electrons are accelerated at an essentially uniform and identical rate: the corresponding curves in Fig. 6 depart only slightly from straight lines, because of weak relativistic effects. As $v_{y} \rightarrow 0$ the slope of these curves is $d v_{y} / d t \simeq 0.06 c \Omega_{\mathrm{e}}$, consistent with the nonrelativistic limit of (4). The untrapped electron trajectory in Fig. 6, in contrast to the two trapped trajectories, is merely a slightly-perturbed Larmor orbit, with no net energy gain.

Figure 7 (a) shows a solution of (6) and (7) for parameters corresponding to those of the simulation with $L=4 \lambda_{0}$. Eight wave modes are included, with amplitudes and wavenumbers approximately equal to those of the modes in Fig. 4 with $\tilde{k} \leqslant 8$. The mode amplitudes are again assumed to be constant. The electron initially lies at the centre of the trapped-particle island corresponding to the first wave excited in the simulation $\left(x=0, v_{x 0}=0.06 c\right)$. The total integration time is $4 \pi / \Omega_{\mathrm{e}}$, i.e. two non-relativistic electron cyclotron periods. The electron remains trapped for long enough to be accelerated in $v_{y}$ (via a modified form of the surfatron process) to 


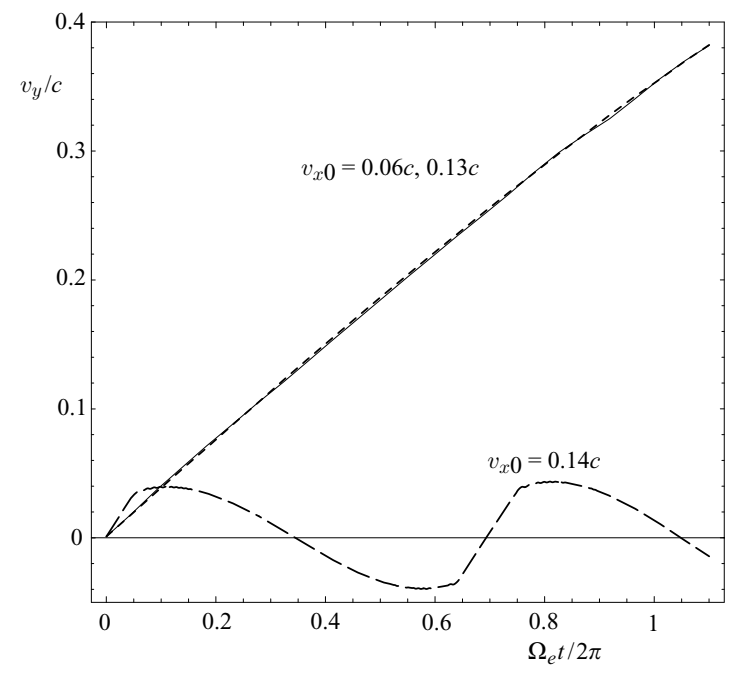

Figure 6. Numerical solutions of equations of motion for parameters corresponding approximately to those of simulation with $L=\lambda_{0}$. The solid curve indicates $v_{y}(t)$ for a trapped electron initially lying close to the separatrix of a trapped-particle island; the short dashed curve is the solution for an electron initially at the centre of the island; the long dashed curve is the solution for an untrapped electron, initially just outside the separatrix (after McClements et al. 2001).

about $0.1 c$. This is only slightly higher than its initial speed in the $x$-direction, and therefore does not represent a very substantial gain in energy. Owing to the presence of a complex time-evolving electric field (arising from the presence of several wave modes), the initially trapped electron has a chaotic trajectory, and for this reason is much more susceptible to detrapping than it would be if only one mode were present. After being detrapped, the electron executes a Larmor gyration before interacting again with the waves. This second interaction results only in a very small energy gain. While individual electrons may remain trapped for a longer period, and thus be accelerated to higher energies, it would appear that the trajectory shown in Fig. 7(a) is more typical. Since the total duration of the simulation with $L=4 \lambda_{0}$ is less than one cyclotron period, it is thus not surprising that strong surfatron acceleration was not observed in that simulation.

In the case of Fig. $7(\mathrm{~b})$ the ratio of wave frequencies to $\Omega_{\mathrm{e}}$ has been increased by a factor of 10 ; the total integration time is again $4 \pi / \Omega_{\mathrm{e}}$. The initial position and velocity of the electron are the same as in Fig. 7 (a) $\left(x=0, v_{x 0}=0.06 c, v_{y 0}=0\right)$. Two phases of surfatron acceleration are apparent. The electron is first accelerated to $v_{y} \simeq 0.15 c$ before it is detrapped. After a partial Larmor gyration, the electron is trapped again and undergoes a second phase of acceleration, up to $v \simeq 0.32 c$, before being detrapped a second time. The final position of the electron is in the bottom right-hand quadrant of the figure. It may be noted that by this time it has not yet completed two complete Larmor orbits. This is partly due to the fact that it is trapped periodically by the waves, and partly because of relativistic time dilation. The key point is that a typical electron is trapped for long enough to be accelerated to energies higher than those found at lower values of $\omega_{\mathrm{pe}} / \Omega_{\mathrm{e}}$. Although the phase space trajectory of a trapped electron is still chaotic, the electron is less susceptible to detrapping because the ratio $E_{0} / c B$ has been increased by a factor 

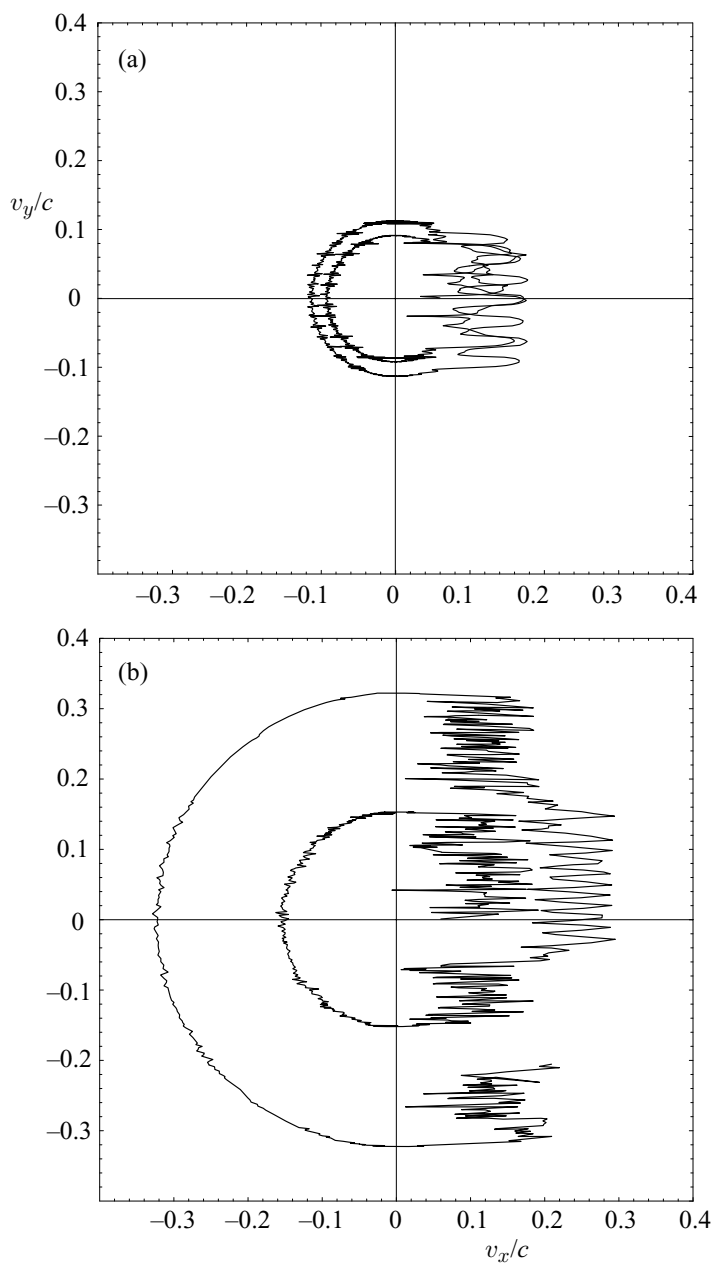

Figure 7. (a) Numerical solution of equations of motion for parameters corresponding to those of simulation with $L=4 \lambda_{0}$. Eight wave modes are included, with amplitudes and wavenumbers approximately equal to those of the dominant modes in Fig. 4. The electron initially lies at the centre of the trapped-particle island corresponding to the first wave excited in the simulation. The total integration time is $4 \pi / \Omega_{\mathrm{e}}$. (b) As (a) except that the ratio of wave frequencies to $\Omega_{\mathrm{e}}$ has been increased by a factor of 10 . The total integration time is again $4 \pi / \Omega_{\mathrm{e}}$ (after McClements et al. 2001).

of ten (cf. (3)). We have again assumed, of course, that the mode amplitudes are constant. In the case of Fig. $7(\mathrm{~b})$, this would require the waves to be present for $2 \times 10^{3}$ electron plasma periods. The actual lifetime of the continuous wave spectrum in the simulation with $L=4 \lambda_{0}$ is not known, since it was still present at the end of the simulation. We note, however, that a similar continuous spectrum observed in one of the unmagnetized Vlasov simulations described by Brunetti et al. (2000) persisted for at least 120 plasma periods, without being driven. In our case, the waves are being driven continuously by an ion beam and thus, ceteris paribus, would be expected to have a longer lifetime than the waves considered by Brunetti and co-workers. 


\section{Summary and conclusions}

We have used a PIC code to carry out a fully self-consistent study of surfatron and stochastic acceleration under conditions similar to those prevailing in the vicinity of SNR shocks. Large amplitude electrostatic waves, required for the surfatron process, are driven via the Buneman instability by ion beams, which are known to be associated with high Mach number quasi-perpendicular shocks. At the end of a simulation with $\omega_{\text {pe }}=100 \Omega_{\mathrm{e}}$, in which wavenumbers $k$ other than that of the initial instability and multiples thereof were suppressed, a small number of electrons trapped by the wave had been accelerated up to about half the speed of light. Direct integration of the electron equations of motion indicates that the acceleration rate is essentially independent of the initial position of the electron within the trapped island region of phase space. However, because most trapped electrons remain close to the separatrix of the island, the trapped electron fraction is extremely sensitive to the wave amplitude, which collapses (partly as a result of the surfatron process itself) after a few tens of plasma periods. A consequence of this is that the efficacy of the surfatron under these conditions is sensitive also to $\omega_{\mathrm{pe}} / \Omega_{\mathrm{e}}$.

In a more realistic simulation, with the same parameters but a larger simulation box size (so that $k$ was not restricted to multiples of the linearly unstable wavenumber), efficient surfatron acceleration was not observed. Shortly after the initial wave reached its peak amplitude, a sequence of sideband instabilities occurred, filling up the available $k$ spectrum and giving rise to a continuum with a power law tail. Electrons were accelerated along the propagation direction of these waves, up to about the phase velocity of the wave with the minimum $k$ permitted by the finite box size. Integration of the equations of motion for several sets of initial conditions, using wave parameters inferred from the simulation results, indicates that surfatron acceleration is ineffective in this case, essentially because electrons are rapidly detrapped when several waves are present. However, we have found that a stochastic version of the surfatron can reappear when $\omega_{\mathrm{pe}} / \Omega_{\mathrm{e}}$ is increased to values greater than $10^{2}$ : if the waves remain in the plasma for at least a few cyclotron periods, electrons can be trapped by them for long enough to undergo acceleration to mildly relativistic energies.

Global one-dimensional PIC simulations of perpendicular collisionless shocks, carried out using artificial mass ratios, largely bear out the results of the local simulations presented here (Schmitz et al. 2002a,b; Hoshino and Shimada 2002). In particular, Schmitz and co-workers observed Langmuir solitons and associated electron phase space holes with different velocities in the shock foot; as in the local analysis, electrons were observed to be accelerated to the highest energies by being successively trapped and untrapped in wave potential wells. Noting that the maximum time available for electron acceleration is of the order of the ion Larmor period $\tau_{\mathrm{Li}}$, Hoshino and Shimada (2002) inferred that electrons could be accelerated to energies of up to $m_{\mathrm{i}} c v_{\mathrm{s}}$, where $m_{\mathrm{i}}$ is the ion mass: for $v_{\mathrm{s}} / c 0.06$, this suggests that electrons with energies of several tens of $\mathrm{MeV}$ could be produced. In the simulations of Schmitz et al. (2002a,b) the shock evolves and reforms on a timescale of around $\tau_{\mathrm{Li}}$ when the plasma beta $(\beta)$ is less than unity. More recent simulations performed using realistic mass ratios by Scholer et al. (2003) confirm that shock reformation occurs at low $\beta$. It should be noted however that the surfatron requires only shockreflected ions, which are present even for $\beta \geqslant 1$, and is not contingent on whether the shock undergoes a process of reformation. Dieckmann et al. (2004a) have carried out 
further studies of electron acceleration at SNR shocks in the local approximation, comparing the PIC and Vlasov methods of simulation, and finding that there is a critical ratio of ion beam speed to initial electron thermal speed above which surfatron acceleration does not occur, due to rapid wave collapse. On the other hand, the lifetime of the saturated wave was found to be considerably longer in the Vlasov case, suggesting that the PIC simulations presented here may underestimate the efficiency of the surfatron. Moreover, the sideband instabilities that potentially limit the effectiveness of the surfatron have been found to be less virulent when the ion beam speeds become mildly relativistic (Dieckmann et al. 2004b).

Of the simulations discussed in the present paper, it is clear that the one with $L=4 \lambda_{0}$ is the more realistic of the two, and the results are more applicable to SNR shocks. Ideally, one would like to carry out simulations with $\omega_{\text {pe }} / \Omega_{\mathrm{e}} \gg 100$, to test the hypothesis that stochastic surfatron acceleration can occur in this regime. This is computationally difficult, however, since the surfatron acceleration timescale scales with $1 / \Omega_{\mathrm{e}}$ while the maximum time step which can be used in the code is determined by $1 / \omega_{\text {pe }}$. It would also be desirable to be able to increase the simulation box size: one would then expect to observe high frequency modes with lower $k$, and hence higher phase speeds. The phase space plot in Fig. 5 suggests that this in turn would lead to the appearance of electrons at higher energies, even without the occurrence of surfatron acceleration. Such a simulation, with a similar or better statistical representation of the electron population, would again place prohibitive demands on computing resources. However, the PIC results and direct numerical solutions of the equations of motion presented in this paper suggest that a combination of stochastic and surfatron acceleration is likely to play an important role in the production of mildly relativistic electrons at SNR shocks. Finally, it is important to note the accumulation, during the past dozen years, of overwhelming experimental evidence for the effectiveness of various particle acceleration processes that exploit large amplitude waves in plasmas. Electron energies approaching $1 \mathrm{GeV}$ have now been achieved using such techniques: for a recent review, we refer to Bingham et al. (2004). These experimental results have validated the associated theoretical and modelling effort, so that the entire field has moved from the conjectural to the concrete. It follows that the scientific basis for invoking such mechanisms in astrophysical contexts, as here, has greatly strengthened in recent years.

\section{Acknowledgements}

The National Supercomputer Centre (NSC) of Sweden provided the computer resources used to carry out this work. It was supported in part by the University of Linköping and the United Kingdom Engineering and Physical Sciences Research Council.

\section{References}

Achterberg, A. and Ball, L. 1994 Astron. Astrophys. 285, 687.

Bell, A. R. 1978 Mon. Not. R. Astron. Soc. 182, 147.

Bernstein, I. B., Greene, J. M. and Kruskal, M. D. 1957 Phys. Rev. 108, 546.

Biermann, P. L. and Cassinelli, J. P. 1993 Astron. Astrophys. 277, 691.

Bingham, R., Mendonça, J. T. and Shukla, P. K. 2004 Plasma Phys. Control. Fusion 46, R1.

Blondin, J. M. and Lundqvist, P. 1993 Astrophys. J. 405, 337. 
Brunetti, M., Califano, F. and Pegoraro, F. 2000 Phys. Rev. E 62, 4109.

Dieckmann, M. E., McClements, K. G., Chapman, S. C., Dendy, R. O. and Drury, L. O'C. 2000a Astron. Astrophys. 356, 377.

Dieckmann, M. E., Ljung, P., Ynnerman, A. and McClements, K. G. 2000b Phys. Plasmas 7,5171 .

Dieckmann, M. E., Eliasson, B., Stathopoulos, A. and Ynnerman, A. 2004a Phys. Rev. Lett. 92, 065006 .

Dieckmann, M. E., Eliasson, B. and Shukla, P. K. 2004b Phys. Plasmas 11, 1394.

Hoshino, M. and Shimada, N. 2002 Astrophys. J. 572, 880.

Karney, C. F. F. 1978 Phys. Fluids 21, 1584.

Katsouleas T. and Dawson, J. M. 1983 IEEE Trans. Nuc. Science NS-30, 3241.

Kruer, W. L., Dawson, J. M. and Sudan, R. N. 1969 Phys. Rev. Lett. 23, 838.

McClements, K. G., Dieckmann, M. E., Ynnerman, A., Chapman, S. C. and Dendy, R. O. 2001 Phys. Rev. Lett. 87, 255002.

Mendonça, J. T. 1996 Physica Scripta T63, 136.

Quest, K. B. 1986 J. Geophys. Res. 91, 8805.

Schmitz, H., Chapman, S. C. and Dendy, R. O. 2002a Astrophys. J. 570, 637.

Schmitz, H., Chapman, S. C. and Dendy, R. O. 2002 b Astrophys. J. 579, 327.

Scholer, M., Shinohara, I. and Matsukiyo, S. 2003 J. Geophys. Res. 108, 1014.

Sckopke, N., Paschmann, G., Bame, S. J., Gosling, J. T. and Russell, C. T. 1983 J. Geophys. Res. 88, 6121.

Shimada, N. and Hoshino, M. 2000 Astrophys. J. Lett. 543, L67.

Tajima, T. and Dawson, J. M. 1979 Phys. Rev. Lett. 43, 267. 